\title{
The value of the tumour-stroma ratio for predicting neoadjuvant chemoradiotherapy response in locally advanced rectal cancer: a case control study
}

Yanting Liang ${ }^{1 \dagger}$, Yaxi Zhu ${ }^{2 \dagger}$, Huan Lin ${ }^{3,4+}$, Shenyan Zhang ${ }^{2 \dagger}$, Suyun $\mathrm{Li}^{3,4}$, Yanqi Huang ${ }^{4,5}$, Chen Liu ${ }^{4,5}$, Jinrong Qu ${ }^{6}$, Changhong Liang ${ }^{4}$, Ke Zhao ${ }^{3,4^{*}}$, Zhenhui Li ${ }^{7^{*}}$ and Zaiyi Liü ${ }^{4^{*}}$

\begin{abstract}
Background: The tumour-stroma ratio (TSR) is recognized as a practical prognostic factor in colorectal cancer. However, TSR assessment generally utilizes surgical specimens. This study aims to investigate whether the TSR evaluated from preoperative biopsy specimens by a semi-automatic quantification method can predict the response after neoadjuvant chemoradiotherapy (nCRT) of patients with locally advanced rectal cancer (LARC).

Methods: A total of 248 consecutive patients diagnosed with LARC and treated with nCRT followed by resection were included. Haematoxylin and eosin (HE)-stained sections of biopsy specimens were collected, and the TSR was evaluated by a semi-automatic quantification method and was divided into three categories, using the cut-offs determined in the whole cohort to balance the proportion of patients in each category. The response to nCRT was evaluated on the primary tumour resection specimen by an expert pathologist using the four-tier tumour regression grade (TRG) system.

Results: The TSR can discriminate patients that are major-responders (TRG 0-1) from patients that are nonresponders (TRG 2-3). Patients were divided into stroma-low (33.5\%), stroma-intermediate (33.9\%), and stroma-high (32.7\%) groups using 56.3 and $72.8 \%$ as the cutoffs. In the stroma-low group, 58 (69.9\%) patients were majorresponders, and only 39 (48.1\%) patients were considered major-responders in the stroma-high group $(P=0.018)$. Multivariate analysis showed that the TSR was the only pre-treatment predictor of response to nCRT (adjusted odds ratio $0.40,95 \%$ confidence interval $0.21-0.76, P=0.002$ ).
\end{abstract}

\footnotetext{
*Correspondence: ksw2024@163.com; lizhenhui621@qq.com;

liuzaiyi@gdph.org.cn

${ }^{\dagger}$ Yanting Liang, Yaxi Zhu, Huan Lin and Shenyan Zhang contributed equally

to this work.

${ }^{3}$ School of Medicine, South China University of Technology, Guangzhou,

China

${ }^{7}$ Department of Radiology, Yunnan Cancer Center, The Third Affiliated

Hospital of Kunming Medical University, Yunnan Cancer Hospital, Kunming

650118, China

${ }^{4}$ Department of Radiology, Guangdong Provincial People's Hospital,

Guangdong Academy of Medical Sciences, 106 Zhongshan Er Road, Guangzhou 510080, China

Full list of author information is available at the end of the article
}

(C) The Author(s). 2021 Open Access This article is licensed under a Creative Commons Attribution 4.0 International License, which permits use, sharing, adaptation, distribution and reproduction in any medium or format, as long as you give appropriate credit to the original author(s) and the source, provide a link to the Creative Commons licence, and indicate if changes were made. The images or other third party material in this article are included in the article's Creative Commons licence, unless indicated otherwise in a credit line to the material. If material is not included in the article's Creative Commons licence and your intended use is not permitted by statutory regulation or exceeds the permitted use, you will need to obtain permission directly from the copyright holder. To view a copy of this licence, visit http://creativecommons.org/licenses/by/4.0/ The Creative Commons Public Domain Dedication waiver (http://creativecommons.org/publicdomain/zero/1.0/) applies to the data made available in this article, unless otherwise stated in a credit line to the data. 
Conclusion: An elevated TSR in preoperative biopsy specimens is an independent predictor of nCRT response in LARC. This semi-automatic quantified TSR could be easily translated into routine pathologic assessment due to its reproducibility and reliability.

Keywords: Whole-slide images, Tumour-stroma ratio, Locally advanced rectal cancer, Neoadjuvant chemoradiotherapy response, Tumour regression grade

\section{Introduction}

Colorectal cancer (CRC) is one of the common causes of cancer-related death worldwide, and approximately onethird of CRC cases occurs in the rectum $[1,2]$. Due to the high risk of locoregional recurrence, neoadjuvant chemoradiotherapy (nCRT) is recommended as the standard therapy for locally advanced rectal cancer (LARC) patients [3]. The addition of nCRT as part of LARC treatment has improved the overall survival (OS) and locoregional relapse-free survival, compared to primary surgery alone. However, due to many factors, including individual differences, tumour size, clinical $\mathrm{T}$ and $\mathrm{N}$ stages, tumour differentiation, treatment-related factors and so on, patients with LARC show varied responses to nCRT due to individual differences. Nearly $20 \%$ of patients show resistance to nCRT, demonstrating minimal regression or even tumour progression [4]. Therefore, it is crucial to predict the therapeutic effect of nCRT for patients before treatment, allowing the selection of LARC patients who would or would not benefit from nCRT, to reduce the immunotoxicity and organ toxicity associated with ineffective nCRT $[5,6]$, and to choose further treatment methods.

Currently, it has become increasingly known that the composition of the tumour microenvironment as well as tumour-stroma interactions play a major role in tumour progression, metastasis, and therapy resistance, leading to poor clinical outcomes [7]. The stroma is one of the key components in the tumour microenvironment. The stromal compartment secretes growth factors and stimulates the formation of new blood vessels to provide the growing tumour with oxygen and nutrients [8]. Several studies have also suggested that the stroma contains more prognostic information than the tumour epithelial component $[9,10]$.

Additionally, there have been different studies that found that the tumour-stroma ratio (TSR) is a valid and practical prognostic factor in solid tumours, including oesophageal cancer, endometrial carcinoma, non-smallcell lung cancer, and CRC [11-14]. Most studies have evaluated the TSR on surgical paraffin sections, while only a few studies have evaluated this parameter on biopsy specimens [7, 15]. A retrospective study from Pelt et al. investigated the value of TSR based on biopsy specimen assessment to predict nCRT response in patients with oesophageal cancer [7]. However, a subjective method was used in their study to evaluate the TSR, with disagreement among pathologists. Currently, digital pathology, especially whole-slide imaging (WSI), is increasingly being used in clinical practice, and is very suitable for full quantitative evaluation of the TSR. It is worthwhile to investigate whether fully quantitative determination of the TSR in preoperative biopsy specimens can predict nCRT efficacy in LARC patients.

Therefore, the objective of this study was to explore whether the TSR evaluated by a semi-automatic quantification method in preoperative biopsy specimens can predict nCRT response in LARC patients, thereby reducing unnecessary pain and cost.

\section{Materials and methods Patients}

A total of 248 consecutive patients diagnosed with clinical stage I-III LARC who underwent nCRT followed by resection at the Sixth Affiliated Hospital of Sun Yat-sen University (SYSU6) between November 2012 and November 2017 were enrolled in the study. The specific inclusion and exclusion criteria are shown in the supplementary material (e.g. Additional file 1). We collected clinicopathologic data from medical records as follows: age, sex, histopathological type, differentiation grade, pre-treatment clinical $\mathrm{T}$ and $\mathrm{N}$ status, pretreatment CEA level, pre-treatment CA199 level, tumour location, time interval between nCRT and surgery, and neoadjuvant radiotherapy dose. All patients received chemoradiotherapy. The combined radiochemotherapy included capecitabine/long-course radiotherapy (45-50 Gy in 25-28 fractions), infusional 5-fluorouracil/longcourse radiotherapy (45-50 Gy in 25-28 fractions), or bolus 5 -fluorouracil/leucovorin/long-course radiotherapy (45-50 Gy in 25-28 fractions). Mono chemotherapy included FOLFOX (folinic acid, fluorouracil and oxaliplatin) or CAPEOX (capecitabine and oxaliplatin). The mono radiotherapy was the regimen of 25 Gy in 5 fractions. The institutional review board of SYSU6 approved this retrospective study (approval no. 2019ZSLYEC-169 date: 2019-06-12), and informed consent was waived.

\section{Tissue slide preparation and scanning}

For each patient, formalin-fixed paraffin-embedded sections of biopsy specimens were cut to a thickness of $4 \mu \mathrm{m}$ and stained with haematoxylin and eosin (HE). 
These slides were scanned by digital whole-slide scanning (Leica, Aperio-AT2, USA) at 40× magnification.

\section{Semi-automatic computation of the TSR}

An expert pathologist (more than 10 years of experience) annotated the tumour epithelium and the whole tumour region (including the tumour epithelium and stroma) on WSIs via ImageScope (version 12.4.3, Leica, USA). To find the stromal region automatically, the following image processing was performed in the MATLAB environment (R2019a, MathWorks, USA): The original image $(40 \times, 0.250 \mu \mathrm{m} / \mathrm{pixel})$ was scaled to a smaller image $(10 \times, 1.00 \mu \mathrm{m} /$ pixel $)$. Then the scaled colour image (RGB-encoded) was converted into a greyscale image, and Gaussian smoothing with a standard deviation of 15 was added. Otsu's method was used to determine the global threshold, segmenting the grey image into the background and tissue region mask (mask $\left.\mathrm{TIS}_{\mathrm{TI}}\right)$. As we already have the annotated tumour epithelium (mask $\mathrm{TUM}_{\mathrm{T}}$ ) and the whole tumour (mask $\mathrm{WHO}_{\mathrm{WH}}$ ) regions, the stroma region (mask $\mathrm{STR}_{\mathrm{STR}}$ was calculated by Boolean operation: mask $_{\mathrm{STR}}=\left(\right.$ mask $_{\mathrm{TUM}} \&$ mask $\left._{\mathrm{TIS}}\right)$ xor $\left(\right.$ mask $_{\mathrm{WHO}}{ }_{\mathrm{TUM}}$ $\&$ mask $\left._{\text {TIS }}\right)$. The calculation process was present in Fig. 1.

The TSR was defined as follows: TSR $=$ stromal area / (tumour epithelial area + stromal area) $\times 100 \%$. A value of $50 \%$ was used to categorize patients into two groups: stroma-high and stroma-low, as determined in previous studies to be the most discriminative [16]. To more accurately stratify patients and develop suitable treatment options for individualized precision therapy, we also adopted a 3-category TSR classification which divided patients into stroma-low, stroma-intermediate, and stroma-high groups, using the cutoffs determined from the whole cohort to balance the proportion of patients in each category. The cutoffs were not defined based on the ROC curve, thus avoiding overfitting of the data.

Since a WSI often takes a few hours to be annotated, another pathologist re-annotated 30 image blocks $(0.5$ $\mathrm{mm} \times 0.5 \mathrm{~mm}$ ) from ten randomly selected WSIs to assess inter-observer variability of the annotation. The selected regions should contain only tumour epithelial

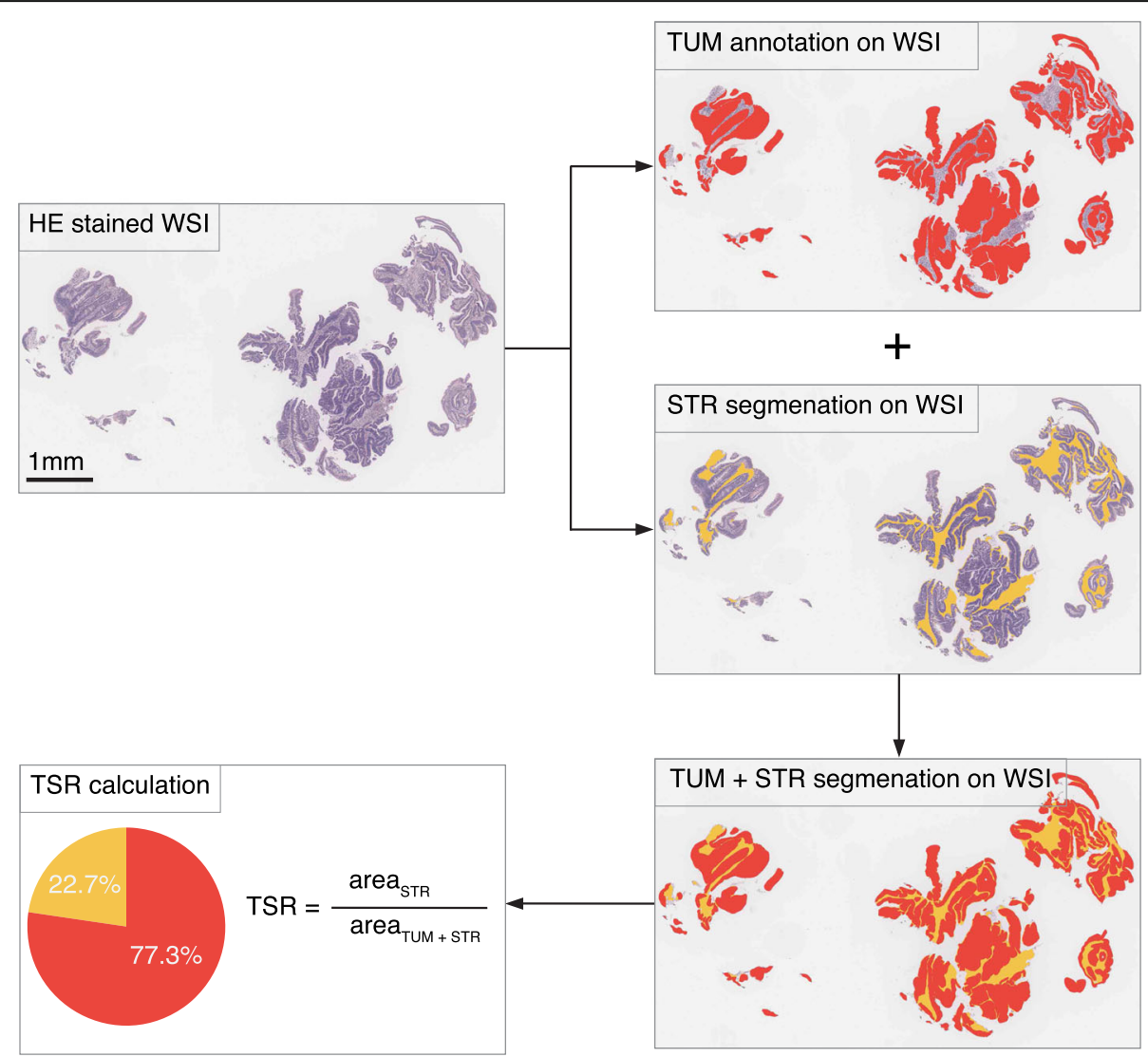

Fig. 1 Semi-automatic tumour-stroma ratio computation workflow. An expert pathologist annotated the tumour epithelium and the whole tumour region (including the tumour epithelium and stroma) on the WSI. The tumour epithelium region is marked in red, and the stroma region is marked in yellow. The stromal and tumour epithelial areas were calculated from the segmentation map. The TSR was defined as the stroma proportion in the sum of the stroma and tumour epithelial areas. TSR, tumour-stroma ratio; WSI, whole-slide image 
and tumour stroma and exclude necrotic or inflammatory regions. The intra-class correlation coefficient (ICC) with 95\% confidence interval (CI) was calculated.

\section{Evaluation of the response to $\mathrm{nCRT}$}

Tumour regression grade (TRG) is significantly associated with disease recurrence and patient survival and can assess the response to nCRT [17]. Therefore we evaluated the response to $\mathrm{nCRT}$ by an expert pathologist using the four-tier TRG system defined by the American Joint Committee on Cancer (AJCC, eighth edition) [18]. The pathologist compared the biopsy and the tumour resection specimen to derive the final TRG. TRG 0 is defined as complete regression with no visible cancer cells and is called a pathological complete response. TRG 1 is characterized by single or small groups of tumour cells. TRG 2 is characterized by residual cancer outgrown by fibrosis. TRG 3 is defined by minimal or no tumour cells killed. The TRG scores were confirmed by pathologic examination after surgical treatment. We categorized the treatment response into two groups. Responses of TRG 0-1 were categorized as major-responders, whereas TRG 2-3 were classified as non-responders.

\section{Statistics}

According to the rule of thumb [19], a minimum of 10 events per variable were necessary for the multivariate analysis. The sample size in our multivariate analysis was sufficient $(n=248)$. All data analyses were conducted in $\mathrm{R}$ software (version 3.6.1). Continuous factors, such as age, were analysed with $t$ tests, while categorical characteristics were tested with Pearson chi-square test or Fisher's exact test. Inter-observer variability was assessed by ICC analysis. The logistic regression model was used to perform univariate and multivariable analyses. Parameters associated with treatment response in univariate analysis were selected for multivariable analysis. In all analyses, a two-tailed $P$ value $<0.05$ was regarded as statistically significant.

\section{Results}

\section{Patient and tumour characteristics}

A total of 248 LARC patients were recruited in this study. The median age was 55 (range, 25 to 79) years, and $176(71 \%)$ were males. Two hundred thirty-two (93.5\%) tumours were moderately or well differentiated, and $16(6.5 \%)$ tumours were poorly differentiated. Table 1 lists the demographics and clinicopathological parameters. The TRG results for patients treated with nCRT were as follows: $27.0 \%$ TRG $0(N=67) ; 31.9 \%$ TRG $1(N=79) ; 37.9 \%$ TRG $2(N=94)$; and $3.2 \%$ TRG 3 $(N=8)$. These results are consistent with most other studies [20, 21]. After dichotomization, 146 patients were categorized as major-responders (TRG 0-1), whereas 102 patients were classified as non-responders (TRG 2-3).

\section{Evaluation of the TSR}

We analysed a total of $248 \mathrm{HE}$-stained biopsy sections from 248 patients. Fig. 2a shows examples of the annotations from two pathologists. Excellent concordance was observed between the two annotations (ICC 0.990, 95\% CI $0.979-0.995$, Fig. 2b). Representative images of stroma-low and stroma-high WSIs and the semiautomatic segmentation results are depicted in Fig. 3.

Using 56.3 and $72.8 \%$ as the cutoffs, the continuous TSR was divided into three categories. Eighty-three patients $(33.5 \%)$ were considered as stroma-low, 84 patients $(33.9 \%)$ as stroma-intermediate, and 81 patients (32.7\%) as stroma-high. When the TSR was separated into two groups using $50 \%$ as the cutoff, a total of 193 (77.8\%) patients were categorized as stroma-high and 55 $(22.2 \%)$ as stroma-low.

\section{The TSR and other predictive factors for treatment response}

Figure. 4a-b shows that the TSR can discriminate majorresponders patients from non-responders, either dichotomous or three-class classification $\left(P<0.05, \chi^{2}\right.$ test). Additionally, the differences were more apparent among the three-class classification. In the stroma-low group, $58(69.9 \%)$ patients were major-responders and only 39 (48.1\%) patients were considered non-responders in the stroma-high group $(P=0.018)$. The distribution of TRG categories versus continuous TSR is shown in Fig. 4c. With the increase in TRG level, the mean value of TSR also increased (except TRG 3). The distribution of major-responders and non-responders versus the continuous TSR is shown in Fig. 4d.

As illustrated in Table 1, there were no statistically significant differences between the major-responder and non-responder groups in most of the clinicopathological characteristics concerning age, sex, histopathological type, differentiation grade, clinical $\mathrm{T} / \mathrm{N}$ status, tumour location, CEA, and CA199 level. By univariate analysis, the time interval to surgery following nCRT > 10 weeks was associated with a greater chance of a response to nCRT (odds ratio [OR] 3.94, 95\% CI 1.16-13.4, $P=$ 0.028 ), but not by multivariate analysis. By multivariate analysis, neoadjuvant radiotherapy doses $\geq 45$ Gy (adjusted OR 3.71, 95\% CI 1.38-10.0, $P=0.002$ ) and receiving the FOLFOX regimen (adjusted OR 2.68, 95\% CI $1.27-5.68, P=0.007)$ were determined to be independent predictors of a good response to nCRT. A high TSR was independently associated with a greater chance of no response to nCRT (adjusted OR 0.40, 95\% CI 0.21-0.76, $P=0.002$, Table 2). 
Table 1 Characteristics of patients in the whole cohort

\begin{tabular}{|c|c|c|c|c|}
\hline & $\begin{array}{l}\text { Total } \\
N=248\end{array}$ & $\begin{array}{l}\text { Major-responders } \\
N=102\end{array}$ & $\begin{array}{l}\text { Non-responders } \\
N=146\end{array}$ & $P$ \\
\hline Age (year, mean $\pm S D$ ) & $54.61 \pm 11.61$ & $54.20 \pm 11.17$ & $55.20 \pm 12.25$ & 0.514 \\
\hline Sex & & & & 0.548 \\
\hline Male & $176(71.0 \%)$ & $101(69.2 \%)$ & $75(73.5 \%)$ & \\
\hline Female & $72(29.0 \%)$ & $45(30.8 \%)$ & $27(26.5 \%)$ & \\
\hline Histopathological type & & & & 0.793 \\
\hline Adenocarcinoma & $236(95.2 \%)$ & $138(94.5 \%)$ & $98(96.1 \%)$ & \\
\hline Mucinous adenocarcinoma & $12(4.8 \%)$ & $8(5.5 \%)$ & $4(3.9 \%)$ & \\
\hline Differentiation grade & & & & 0.274 \\
\hline Well or moderate & $232(93.5 \%)$ & $134(91.8 \%)$ & $98(96.1 \%)$ & \\
\hline Poor & $16(6.5 \%)$ & $12(8.2 \%)$ & $4(3.9 \%)$ & \\
\hline CT status & & & & 0.657 \\
\hline cT2 & $10(4.0 \%)$ & $6(4.1 \%)$ & $4(4.0 \%)$ & \\
\hline cT3 & $175(70.6 \%)$ & $106(72.6 \%)$ & $69(67.6 \%)$ & \\
\hline cT4 & $63(25.4 \%)$ & $34(23.3 \%)$ & $29(28.4 \%)$ & \\
\hline cN status & & & & 0.07 \\
\hline $\mathrm{CNO}$ & $38(15.3 \%)$ & 19 (13.0\%) & 19 (18.6\%) & \\
\hline $\mathrm{cN} 1$ & $101(40.7 \%)$ & $54(37.0 \%)$ & $47(46.1 \%)$ & \\
\hline $\mathrm{cN} 2$ & $109(44.0 \%)$ & $73(50.0 \%)$ & $36(35.3 \%)$ & \\
\hline Tumour location & & & & 0.787 \\
\hline$<5 \mathrm{~cm}$ & $140(56.5 \%)$ & $85(58.2 \%)$ & $55(53.9 \%)$ & \\
\hline $5-10 \mathrm{~cm}$ & $103(41.5 \%)$ & $58(39.7 \%)$ & $45(44.1 \%)$ & \\
\hline$>10 \mathrm{~cm}$ & $5(2.0 \%)$ & $3(2.1 \%)$ & $2(2.0 \%)$ & \\
\hline CEA level & & & & 0.731 \\
\hline Normal & $143(57.7 \%)$ & $86(58.9 \%)$ & $57(55.9 \%)$ & \\
\hline Abnormal & $105(42.3 \%)$ & $60(41.1 \%)$ & $45(44.1 \%)$ & \\
\hline CA199 level & & & & 0.550 \\
\hline Normal & $196(79.0 \%)$ & $113(77.4 \%)$ & $83(81.4 \%)$ & \\
\hline Abnormal & $52(21.0 \%)$ & $33(22.6 \%)$ & $19(18.6 \%)$ & \\
\hline Chemotherapeutic regimen & & & & 0.001 \\
\hline 5-fluorouracil & $35(14.1 \%)$ & $16(11.0 \%)$ & $19(18.6 \%)$ & \\
\hline Capecitabine & $33(13.3 \%)$ & $10(6.8 \%)$ & $23(22.5 \%)$ & \\
\hline FOLFOX & $150(60.5 \%)$ & $104(71.2 \%)$ & $46(45.1 \%)$ & \\
\hline Other regimens & $30(12.1 \%)$ & $16(11.0 \%)$ & $14(81.4 \%)$ & \\
\hline Time interval between nCRT and surgery & & & & 0.046 \\
\hline$<7$ weeks & $13(5.3 \%)$ & $4(2.8 \%)$ & $9(8.8 \%)$ & \\
\hline $7-10$ weeks & $91(36.8 \%)$ & $50(34.5 \%)$ & $41(40.2 \%)$ & \\
\hline$>10$ weeks & $143(57.9 \%)$ & $91(62.8 \%)$ & $52(51.0 \%)$ & \\
\hline Neoadjuvant radiotherapy dose & & & & 0.012 \\
\hline$<45$ Gy & $20(8.1 \%)$ & $6(4.1 \%)$ & $14(13.7 \%)$ & \\
\hline$\geq 45 \mathrm{~Gy}$ & $228(91.9 \%)$ & $140(95.9 \%)$ & $88(86.3 \%)$ & \\
\hline
\end{tabular}


a

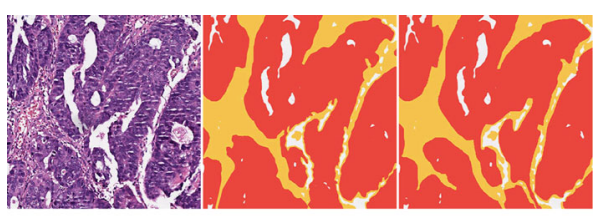

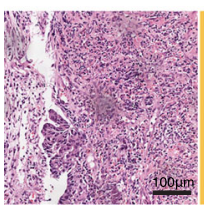

Image

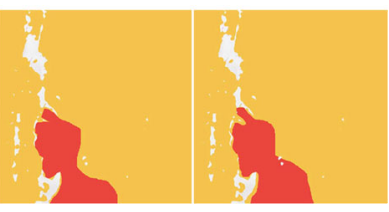

Annotation 1 Annotation 2 b

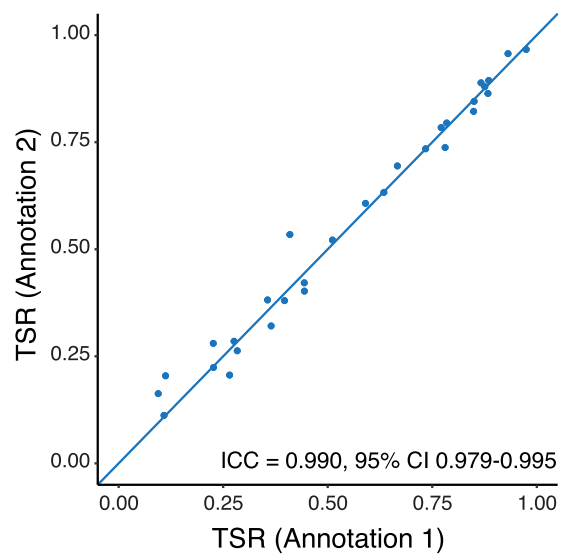

Fig. 2 Consistency analysis of inter-observer variability of the annotations of two pathologists. (a) Examples of two pathologists' annotations. (b) Excellent concordance was observed between the two annotations

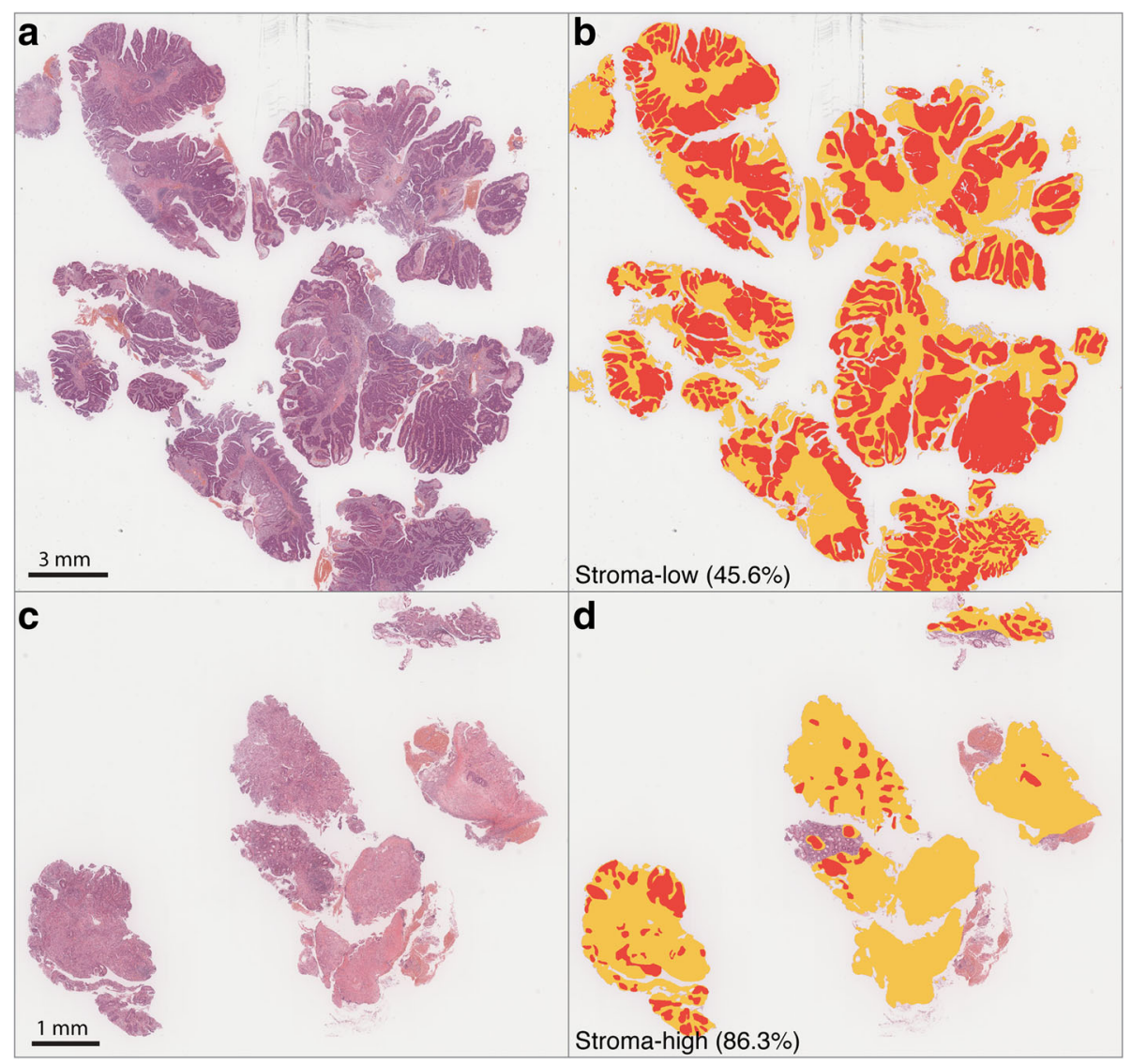

Fig. 3 Representative images of stroma-low and stroma-rich tumour biopsies. (a, c) HE-stained biopsy sections of LARC with low TSR and high TSR, respectively. (b, d) The same regions segmented by a semi-automatic method for calculation of the TSR. HE, haematoxylin and eosin; LARC, locally advanced rectal cancer; TSR, tumour-stroma ratio 


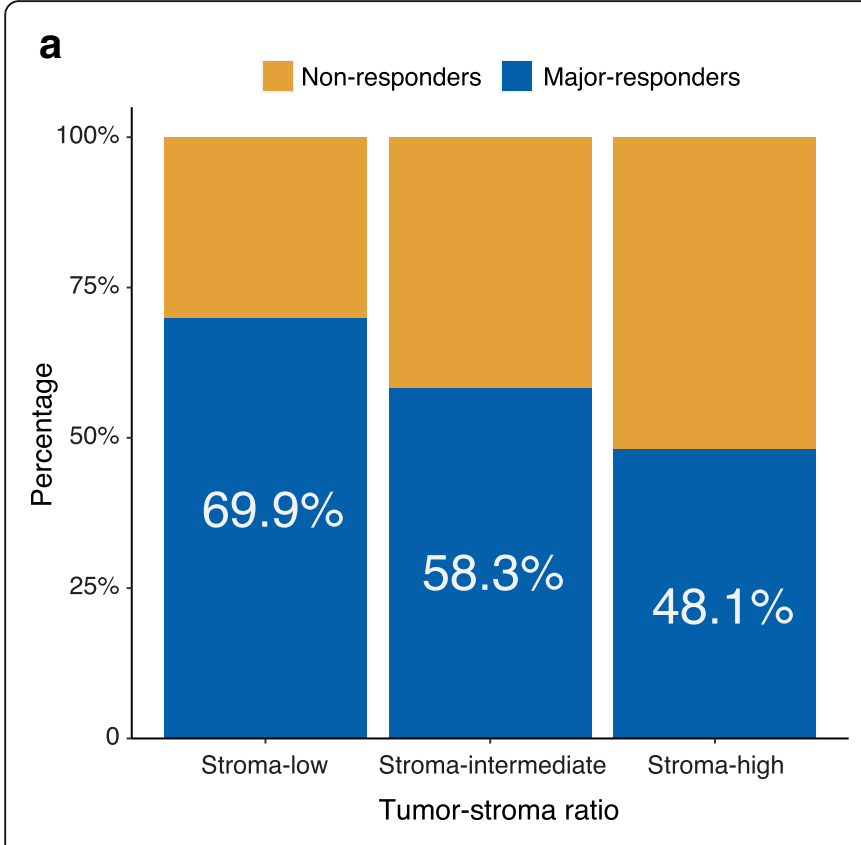

b

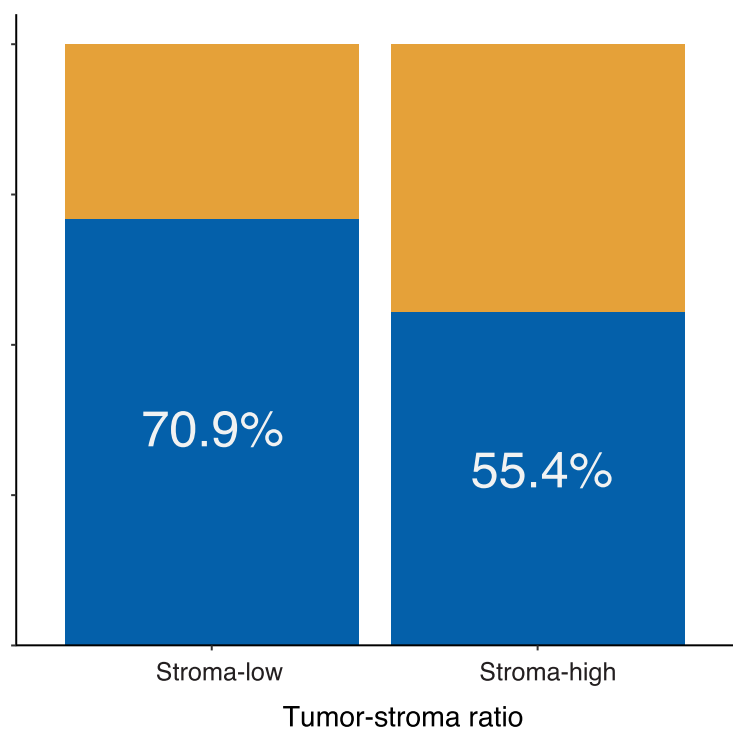

C

d
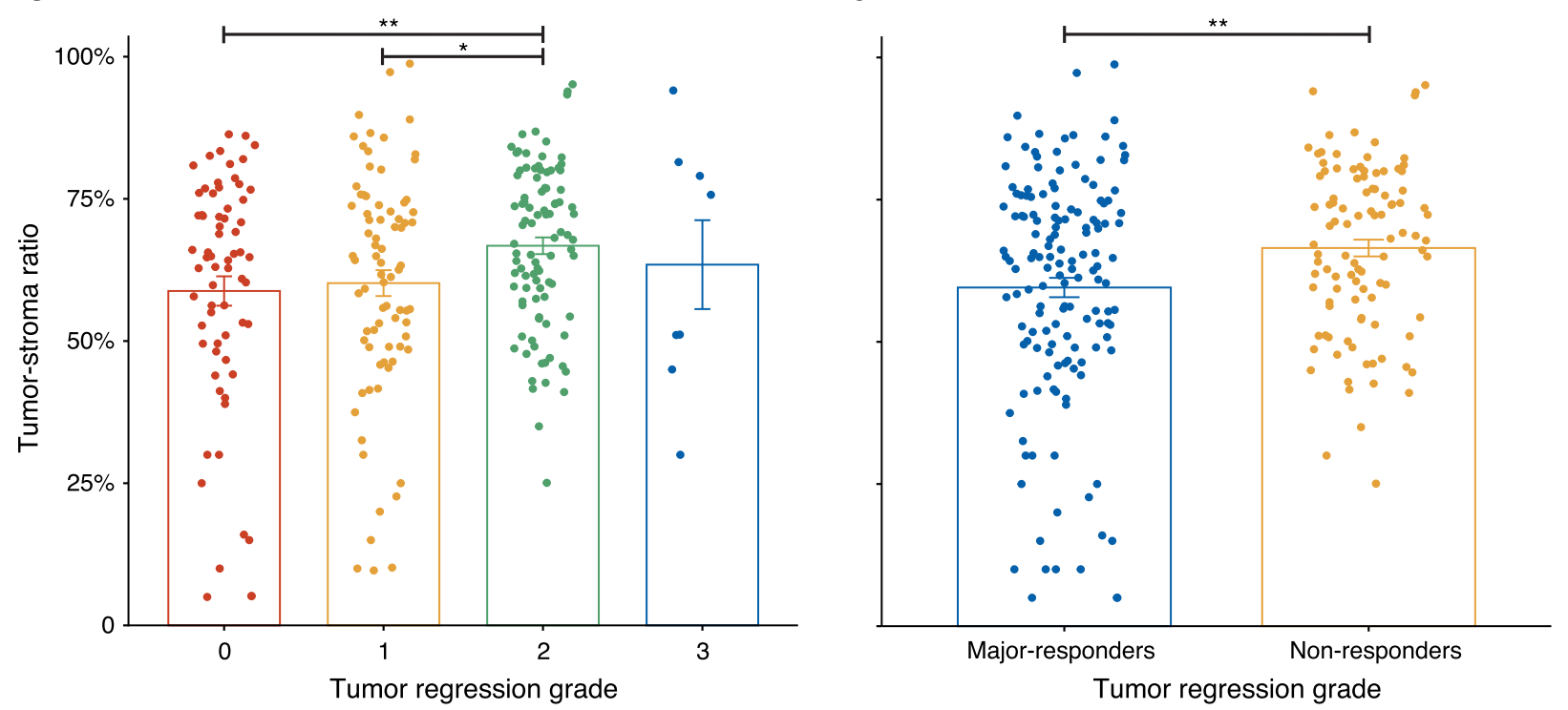

Fig. 4 TSR can discriminate patients as major responders (TRG 0-1) from non-responders (TRG 2-3). (a) The distribution of major responders (in blue) and non-responders (in yellow) within the 3-category TSR. (b) The distribution of major responders (in blue) and non-responders (in yellow) within two stroma categories. (c) The distribution of TRG categories versus the continuous TSR. (d) The distribution of major responders and nonresponders versus the continuous TSR. TRG, tumour regression grade; TSR, tumour-stroma ratio

\section{Discussion}

In this study, we evaluated the TSR by a semi-automatic quantification method from preoperative biopsy specimens is the hope of selecting patients with LARC who would or would not benefit from nCRT. Patients with high amounts of stroma have a significantly superior chance of not responding to nCRT (TRG 2-3) compared with patients with low stroma.

Tumour regression grade is an important approach to assess the response to nCRT in LARC patients [22], which is assessed by a histopathologist largely based on the proportion of residual tumour cells and fibrosis. It is possible that tumours with a high stromal content may be misclassified as showing a greater degree of regression than the reality due to the desmoplastic stromal reaction. However, it is notable that the amount of stroma is inversely correlated with the degree of tumour regression in our research, which is contrary to the above inference. This further demonstrates that there is an association between TSR and tumour regression grade. 
Table 2 Uni- and multivariable analyses with the logistic regression

\begin{tabular}{|c|c|c|c|c|}
\hline & Univariable anal & & Multivariable an & \\
\hline & OR $(95 \% \mathrm{Cl})$ & $P$ & AOR $(95 \% \mathrm{Cl})$ & $P$ \\
\hline Age (year, mean $\pm S D)$ & $0.99(0.97-1.01)$ & 0.505 & & \\
\hline Sex & & & & \\
\hline Male & Ref & & & \\
\hline Female & $0.81(0.46-1.42)$ & 0.458 & & \\
\hline Histopathological type & & & & \\
\hline Adenocarcinoma & Ref & & & \\
\hline Mucinous adenocarcinoma & $1.42(0.42-4.85)$ & 0.575 & & \\
\hline Differentiation grade & & & & \\
\hline Well or moderate & Ref & & & \\
\hline Poor & $2.19(0.69-7.01)$ & 0.185 & & \\
\hline CT status & & & & \\
\hline cT2 & Ref & & & \\
\hline cT3 & $1.02(0.28-3.76)$ & 0.971 & & \\
\hline cT4 & $0.78(0.20-3.04)$ & 0.722 & & \\
\hline $\mathrm{cN}$ status & & & & \\
\hline cNO & Ref & & & \\
\hline $\mathrm{cN} 1$ & $1.15(0.54-2.42)$ & 0.715 & & \\
\hline $\mathrm{cN} 2$ & $2.03(0.96-4.30)$ & 0.065 & & \\
\hline Tumour location & & & & \\
\hline$<5 \mathrm{~cm}$ & Ref & & & \\
\hline $5-10 \mathrm{~cm}$ & $0.83(0.50-1.40)$ & 0.491 & & \\
\hline$>10 \mathrm{~cm}$ & $0.97(0.16-6.00)$ & 0.974 & & \\
\hline CEA level & & & & \\
\hline Normal & Ref & & & \\
\hline Abnormal & $0.88(0.53-1.47)$ & 0.636 & & \\
\hline CA199 level & & & & \\
\hline Normal & Ref & & & \\
\hline Abnormal & $1.28(0.68-2.40)$ & 0.450 & & \\
\hline Time interval between $\mathrm{nCRT}$ & & & & \\
\hline$<7$ weeks & Ref & & & \\
\hline $7-10$ weeks & $2.74(0.79-9.56)$ & 0.113 & & \\
\hline$>10$ weeks & $3.94(1.16-13.4)$ & 0.028 & & \\
\hline Chemotherapeutic regimen & & & & \\
\hline 5-fluorouracil & Ref & & Ref & \\
\hline Capecitabine & $0.52(0.19-1.40)$ & 0.194 & $0.45(0.16-1.28)$ & 0.134 \\
\hline FOLFOX & $2.68(1.27-5.68)$ & 0.010 & $2.93(1.33-6.46)$ & 0.007 \\
\hline Other regimens & $1.36(0.51-3.61)$ & 0.541 & $1.53(0.55-4.28)$ & 0.415 \\
\hline Neoadjuvant radiotherapy d & & & & \\
\hline$<45$ Gy & Ref & & Ref & \\
\hline$\geq 45 \mathrm{~Gy}$ & $3.71(1.38-10.0)$ & 0.006 & $5.25(1.84-14.9)$ & 0.002 \\
\hline TSR (three categery) & & & & \\
\hline Low & Ref & & Ref & \\
\hline Intermediate & $0.60(0.32-1.14)$ & 0.121 & $0.45(0.22-0.90)$ & 0.025 \\
\hline High & $0.40(0.21-0.76)$ & 0.005 & $0.32(0.16-0.65)$ & 0.002 \\
\hline
\end{tabular}


The more stroma there is, the lower the degree of tumour regression, which indicates a poorer prognosis. This result is consistent with other studies showing that a higher TSR is associated with a worse prognosis [23, 24].

Most of the previous literature has studied the TSR based on surgical specimens [13, 14, 25, 26]. Our study evaluated TSR on preoperative diagnostic biopsy sections of rectal cancer. Likewise, in digestive tract tumours, the results of oesophageal cancer research are consistent with our research $[7,15]$. They found that the TSR can predict the response to nCRT in oesophageal cancer patients, but they assessed the TSR in artificially selected areas by visually subjective semi-quantitative scoring $(10,20 \%, \ldots, 100 \%)$. Our study adopted a full quantification method on WSIs in LARC patients. Compared with the results from Pelt et al. (Kappa $=0.73)$ [7], our method achieved a higher degree of agreement for TSR evaluation $(\mathrm{ICC}=0.99)$. Furthermore, the multivariate analysis uncovered that only the TSR, neoadjuvant radiotherapy dose and FOLFOX regimen were independent predictive factors. However, the two latter variables are markers in treatment, and only TSR is a pretreatment predictor. From the perspective of changing treatment schemes, preoperative assessment of the TSR is more likely to guide adaptive treatment planning for LARC patients. Owing to the differences in the areas evaluated for TSR in biopsy specimens and surgical specimens, the use of $50 \%$ as the cutoff which has been reported in the preceding literature may not be applicable to biopsy specimens. The tertile approach used in our study may be more appropriate for evaluation of the TSR of biopsy specimens.

There are also studies that use preoperative biopsy HE histology image analysis to predict the nCRT response of LARC patients. For example, Zhang et al. obtained quantitative features of preoperative biopsy HE-stained histology slides through machine learning and investigated its capability in predicting the treatment response of patients to nCRT [27]. Nevertheless, these signatures lack biological interpretability and are not easily translated into routine pathologic assessments. In our study, the relation between the TSR and the response to nCRT may be explained pathophysiologically. Extensive research has shown that the stroma plays a major role in the induction of chemoradiotherapy resistance [28]. For instance, chemoradiotherapy-induced damage in the tumour environment induces stromal cell stress, which in turn secretes additional factors that accelerate cancer cell proliferation, survival, invasion, and metastasis [29]. Therefore, our outcome that patients with high amounts of stroma are more likely to be related to poor response to $\mathrm{nCRT}$ has good interpretability.
Our findings did not show a significant difference in pathological complete response (pCR: TRG 0 vs. TGR 1-3) between the stroma-high group and the stroma-low group, but we found a significant difference between majorresponders and non-responders. Additionally, as the tumour regression grade increased, the stroma content showed an upward trend (TRG 3 was not included due to the small number of cases). The lack of a pCR difference might be due to the small number of TRG 0 cases (27.0\%). LARC patients with pCR may choose a "watch \& wait" policy [30]. However, the main objective of this article was to select LARC patients who could benefit from nCRT, so it may be more meaningful to study patients who obtain good responses.

To our knowledge, no published studies to date have explored the relationship between the TSR and the response after nCRT in LARC patients. Unlike other studies [26], this is the first to assess the relative amounts of tumour and stroma by a semi-automatic method from preoperative diagnostic biopsy sections. The present study shows that patients with more stromata are less likely to benefit from nCRT. This might indicate that patients with high amounts of stroma that will probably not respond to nCRT, should adjust their therapeutic strategy. For instance, these tumours could receive therapies targeting stromal elements in combination with cytotoxic drugs. Another alternative is that they might be able to proceed directly to radical surgery without the use of neoadjuvant chemoradiotherapy, thereby avoiding exposure to the side effects of chemoradiation treatment. However, these conclusions need to be further validated in long-term, large-sample prospective studies.

Our study had several limitations. First, this was a retrospective study from a single center. The number of cases was too small to conduct validation. Further multicenter and prospective studies in a large cohort are needed in the future before implementing the TSR in routine clinical practice. Second, the TRG was used as the endpoint outcome as the patient's DFS and OS information was not available. In addition, in the current study, we used a semi-automatic method to evaluate the TSR, which retained some subjective deviations and required manual annotation. However, based on the images annotated in this paper, we will develop an artificial intelligence based method to perform pixel-level tumour-stroma segmentation in future research.

In conclusion, this study introduced a semi-automatic method to quantify the TSR and proved that it is an important parameter to predict the response of patients with LARC who underwent nCRT. This might indicate that the TSR may potentially be a powerful tool to identify patients who will benefit from nCRT, thereby guiding the clinicians to select suitable management strategies and adjusting the treatment methods in a timely manner to minimize side effects and medical expenses. 


\section{Abbreviations}

CRC: Colorectal cancer; nCRT: Neoadjuvant chemoradiotherapy; LARC: Locally advanced rectal cancer; OS: Overall survival; TSR: Tumour-stroma ratio; WSI: Whole-slide image; SYSU6: Sixth Affiliated Hospital of Sun Yat-sen University; HE: Haematoxylin and eosin; ICC: Intra-class correlation coefficient; Cl: Confidence interval; TRG: Tumour regression grade; pCR: Pathological complete response

\section{Supplementary Information}

The online version contains supplementary material available at https://doi. org/10.1186/s12885-021-08516-x.

Additional file 1: Supplementary Methods.

\section{Acknowledgements}

Not applicable.

\section{Authors' contributions}

YTL and YXZ were responsible for the study design. SYL, YQH, CL and JRQ were responsible for formal analysis and investigation and data curation. KZ were responsible for data analysis. $\mathrm{HL}$ and $\mathrm{SYZ}$ were responsible for manuscript drafting. ZYL, CHL and ZHL provided discussion, critical feedback and manuscript editing. All authors approved the study protocol and they read and approved the final manuscript.

\section{Funding}

This work was supported by the National Key Research and Development Program of China [2017YFC1309102], National Science Fund for Distinguished Young Scholars [81925023], National Natural Science Foundation of China [82001986, 81771912 and 82071892], and High-level Hospital Construction Project [DFJH201805 and DFJH201914].

\section{Availability of data and materials}

The datasets used and/or analyzed during the current study are not publicly available due to personal information involved but are available from the corresponding author on reasonable request.

\section{Declarations}

\section{Ethics approval and consent to participate}

The study was performed in accordance with the Declaration of Helsinki. The institutional review board of the Sixth Affiliated Hospital of Sun Yat-sen University approved this retrospective study (approval no. 2019ZSLYEC-169 date: 2019-06-12), and the informed consent was waived by the same ethics committee that approved the study (Sixth Affiliated Hospital of Sun Yat-sen University).

\section{Consent for publication}

Not applicable.

\section{Competing interests}

The authors declared no conflicts of interest.

\footnotetext{
Author details

${ }^{1}$ Guangdong Cardiovascular Institute, Guangdong Provincial People's Hospital, Guangdong Academy of Medical Sciences, Guangzhou, China. ${ }^{2}$ Department of Pathology, The Sixth Affiliated Hospital of Sun Yat-sen University, Guangzhou, China. ${ }^{3}$ School of Medicine, South China University of Technology, Guangzhou, China. ${ }^{4}$ Department of Radiology, Guangdong Provincial People's Hospital, Guangdong Academy of Medical Sciences, 106 Zhongshan Er Road, Guangzhou 510080, China. ${ }^{5}$ The Second School of Clinical Medicine, Southern Medical University, Guangzhou, China. ${ }^{6}$ Department of Radiology, The Affiliated Cancer Hospital of Zhengzhou University \& Henan Cancer Hospital, Zhengzhou, China. ${ }^{7}$ Department of Radiology, Yunnan Cancer Center, The Third Affiliated Hospital of Kunming Medical University, Yunnan Cancer Hospital, Kunming 650118, China.
}

Received: 19 April 2021 Accepted: 16 June 2021

Published online: 25 June 2021

\section{References}

1. Dekker E, Tanis PJ, Vleugels JLA, Kasi PM, Wallace MB. Colorectal cancer. Lancet. 2019;394(10207):1467-80. https://doi.org/10.1016/S0140-6736(1 9)32319-0.

2. Siegel RL, Miller KD, Goding Sauer A, Fedewa SA, Butterly LF, Anderson JC, et al. Colorectal cancer statistics, 2020. CA Cancer J Clin. 2020;70(3):145-64. https://doi.org/10.3322/caac.21601.

3. Benson AB, Venook AP, Al-Hawary MM, Cederquist L, Chen YJ, Ciombor KK, et al. Rectal cancer, version 2.2018, NCCN clinical practice guidelines in oncology. J Natl Compr Cancer Netw. 2018;16(7):874-901. https://doi.org/10. 6004/jnccn.2018.0061.

4. Dayde D, Tanaka I, Jain R, Tai MC, Taguchi A. Predictive and prognostic molecular biomarkers for response to neoadjuvant chemoradiation in rectal cancer. Int J Mol Sci. 2017;18(3). https://doi.org/10.3390/ijms18030573.

5. Peng X, Wei Z, Gerweck LE. Making radiation therapy more effective in the era of precision medicine. Precision Clin Med. 2020;3(4):272-83. https://doi. org/10.1093/pcmedi/pbaa038.

6. Zou B, Schuster JP, Niu K, Huang Q, Rühle A, Huber PE. Radiotherapyinduced heart disease: a review of the literature. Precision Clin Med. 2019; 2(4):270-82. https://doi.org/10.1093/pcmedi/pbz025.

7. van Pelt GW, Krol JA, Lips IM, Peters FP, van Klaveren D, Boonstra JJ, et al. The value of tumour-stroma ratio as predictor of pathologic response after neoadjuvant chemoradiotherapy in esophageal cancer. Clin Transl Radiat Oncol. 2020;20:39-44. https://doi.org/10.1016/j.ctro.2019.11.003.

8. Banerjee S, Ghosh T, Barik S, Das A, Ghosh S, Bhuniya A, et al. Neem leaf glycoprotein prophylaxis transduces immune dependent stop signal for tumour angiogenic switch within tumour microenvironment. PLoS One. 2014;9(11):e110040. https://doi.org/10.1371/journal.pone.0110040.

9. Beck AH, Sangoi AR, Leung S, Marinelli RJ, Nielsen TO, van de Vijver MJ, et al. Systematic analysis of breast cancer morphology uncovers stromal features associated with survival. Sci Transl Med. 2011;3(108):108ra113. https://doi.org/10.1126/scitranslmed.3002564.

10. Kather JN, Krisam J, Charoentong P, Luedde T, Herpel E, Weis C-A, et al. Predicting survival from colorectal cancer histology slides using deep learning: a retrospective multicenter study. PLoS Med. 2019;16(1):e1002730. https://doi.org/10.1371/journal.pmed.1002730.

11. Zhao K, Li Z, Yao S, Wang Y, Wu X, Xu Z, et al. Artificial intelligence quantified tumour-stroma ratio is an independent predictor for overall survival in resectable colorectal cancer. EBioMedicine. 2020;61:103054. https://doi.org/10.1016/j.ebiom.2020.103054.

12. Wang $\mathrm{K}, \mathrm{Ma}$ W, Wang J, Yu L, Zhang $X$, Wang Z, et al. Tumour-stroma ratio is an independent predictor for survival in esophageal squamous cell carcinoma. J Thorac Oncol. 2012;7(9):1457-61. https://doi.org/10.1097/JTO. Ob013e318260dfe8.

13. Zhang $T, X u J$, Shen H, Dong W, Ni Y, Du J. Tumour-stroma ratio is an independent predictor for survival in NSCLC. Int J Clin Exp Pathol. 2015;8(9): 11348-55.

14. Panayiotou H, Orsi NM, Thygesen HH, Wright Al, Winder M, Hutson R, et al. The prognostic significance of tumour-stroma ratio in endometrial carcinoma. BMC Cancer. 2015;15(1):955. https://doi.org/10.1186/s12885-01 5-1981-7.

15. Hale MD, Nankivell M, Hutchins GG, Stenning SP, Langley RE, Mueller W, et al. Biopsy proportion of tumour predicts pathological tumour response and benefit from chemotherapy in resectable oesophageal carcinoma: results from the UK MRC OE02 trial. Oncotarget. 2016;7(47):77565-75. https://doi.org/10.18632/oncotarget.12723.

16. van Pelt GW, Sandberg TP, Morreau H, Gelderblom H, van Krieken JHJM, Tollenaar RAEM, et al. The tumour-stroma ratio in colon cancer: the biological role and its prognostic impact. Histopathology. 2018;73(2):197206. https://doi.org/10.1111/his.13489.

17. Trakarnsanga A, Gönen M, Shia J, Nash GM, Temple LK, Guillem JG, et al. Comparison of tumour regression grade systems for locally advanced rectal cancer after multimodality treatment. J Natl Cancer Inst. 2014;106(10). https://doi.org/10.1111/his.13489.

18. Kim SH, Chang HJ, Kim DY, Park JW, Baek JY, Kim SY, et al. What is the ideal tumour regression grading system in rectal cancer patients after preoperative chemoradiotherapy? Cancer Res Treat. 2016;48(3):998-1009. https://doi.org/10.4143/crt.2015.254. 
19. Peduzzi P, Concato J, Kemper E, Holford TR, Feinstein AR. A simulation study of the number of events per variable in logistic regression analysis. J Clin Epidemiol. 1996;49(12):1373-9. https://doi.org/10.1016/50895-4356(96)0023 6-3.

20. Zeng W-G, Liang J-W, Wang Z, Zhang X-M, Hu J-J, Hou H-R, et al. Clinical parameters predicting pathologic complete response following neoadjuvant chemoradiotherapy for rectal cancer. Chin J Cancer. 2015;34(10):468-74. https://doi.org/10.1186/s40880-015-0033-7.

21. Yoon SM, Kim DY, Kim TH, Jung KH, Chang HJ, Koom WS, et al. Clinical parameters predicting pathologic tumour response after preoperative chemoradiotherapy for rectal cancer. Int J Radiat Oncol Biol Phys. 2007; 69(4):1167-72. https://doi.org/10.1016/j.jijobp.2007.04.047.

22. Nagtegaal ID, Glynne-Jones R. How to measure tumour response in rectal cancer? An explanation of discrepancies and suggestions for improvement. Cancer Treat Rev. 2020:84:101964. https://doi.org/10.1016/..ctrv.2020.101964.

23. Eriksen AC, Sørensen FB, Lindebjerg J, Hager H, dePont Christensen R, KjærFrifeldt $\mathrm{S}$, et al. The prognostic value of tumour stroma ratio and tumour budding in stage II colon cancer. A nationwide population-based study. Int J Color Dis. 2018;33(8):1115-24. https://doi.org/10.1007/s00384-018-3076-9.

24. Vogelaar FJ, van Pelt GW, van Leeuwen AM, Willems JM, Tollenaar RAEM, Liefers GJ, et al. Are disseminated tumour cells in bone marrow and tumour-stroma ratio clinically applicable for patients undergoing surgical resection of primary colorectal cancer? The Leiden MRD study. Cell Oncol (Dordr). 2016;39(6):537-44. https://doi.org/10.1007/s13402-016-0296-2.

25. Downey $C L$, Simpkins SA, White J, Holliday DL, Jones JL, Jordan LB, et al. The prognostic significance of tumour-stroma ratio in oestrogen receptorpositive breast cancer. Br J Cancer. 2014;110(7):1744-7. https://doi.org/10.1 038/bjc.2014.69.

26. Zunder SM, van Pelt GW, Gelderblom HJ, Mancao C, Putter H, Tollenaar RA, et al. Predictive potential of tumour-stroma ratio on benefit from adjuvant bevacizumab in high-risk stage II and stage III colon cancer. Br J Cancer. 2018;119(2):164-9. https://doi.org/10.1038/s41416-018-0083-0.

27. Zhang F, Yao S, Li Z, Liang C, Zhao K, Huang Y, et al. Predicting treatment response to neoadjuvant chemoradiotherapy in local advanced rectal cancer by biopsy digital pathology image features. Clin Transl Med. 2020; 10(2):e110. https://doi.org/10.1002/ctm2.110.

28. Valkenburg KC, de Groot AE, Pienta KJ. Targeting the tumour stroma to improve cancer therapy. Nat Rev Clin Oncol. 2018;15(6):366-81. https://doi. org/10.1038/s41571-018-0007-1.

29. Hu Y, Yan C, Mu L, Huang K, Li X, Tao D, et al. Fibroblast-derived exosomes contribute to chemoresistance through priming cancer stem cells in colorectal cancer. PLoS One. 2015;10(5):e0125625. https://doi.org/10.1371/ journal.pone.0125625.

30. Fernandez LM, São Julião GP, Figueiredo NL, Beets GL, van der Valk MJM, Bahadoer RR, et al. Conditional recurrence-free survival of clinical complete responders managed by watch and wait after neoadjuvant chemoradiotherapy for rectal cancer in the International Watch \& Wait Database: a retrospective, international, multicentre registry study. Lancet Oncol. 2021;22(1):43-50. https://doi.org/10.1016/s1470-2045(20)30557-x.

\section{Publisher's Note}

Springer Nature remains neutral with regard to jurisdictional claims in published maps and institutional affiliations.

Ready to submit your research? Choose BMC and benefit from:
- fast, convenient online submission
- thorough peer review by experienced researchers in your field
- rapid publication on acceptance
- support for research data, including large and complex data types
- gold Open Access which fosters wider collaboration and increased citations
- maximum visibility for your research: over 100M website views per year
At BMC, research is always in progress.
Learn more biomedcentral.com/submissions

